\title{
Role of surfaces and interfaces in mechanical and piezoresistive properties of bare Si/Ge heterostructure nanowires
}

\section{Shuang-ying Lei, 'Shan Luan, Yi Li, Hong Yu, and Qing-An Huang}

Key Laboratory of Microelectromechanical Systems of the Ministry of Education, Southeast University, Nanjing 210096, China

E-mail address: 1sy@seu.edu.cn

\begin{abstract}
Mechanical and piezoresistive properties of bare $\mathrm{Si}_{3-x} / \mathrm{Ge}_{x}$ heterostructure nanowires (HNWs) have been investigated by using first-principles calculations. It is found that Young's modulus of $\mathrm{Si}_{2} / \mathrm{Ge}_{1} \mathrm{HNW}$ is much smaller than that of other $\mathrm{Si}_{3-x} / \mathrm{Ge}_{x}$, attributed to the partial surface reconstruction. Moreover, $\mathrm{Si}_{3-x} / \mathrm{Ge}_{x} \mathrm{HNWs}$ with clear interfaces exhibit semiconductor-like band, while those with mixed interfaces are of metal-like band. Enhanced piezoresistive coefficients can be obtained for HNWs with clear interfaces, as compared with those with mixed interfaces. Our results indicate that bare $\mathrm{Si}_{3-x} / \mathrm{Ge}_{x} \mathrm{HNWs}$ with clear interfaces have potential applications as pressure sensors due to the enhanced piezoresistance.
\end{abstract}




\section{Introduction}

The complete piezoresistive tensor for Si was determined experimentally through applying uniaxial tensile stress on single-crystal rods by Smith in 1954 [1], where the electron transfer effect between energy bands was proposed to be responsible for the piezoresistive effect. Since then, piezoresistive effect of the one-dimensional Si structure has been extensively studied to pursue the functional devices for micro-electromechanical systems, such as stress sensor [2], accelerometer [3], and gyroscope [4]. With the rapid development of technique on micro-electromechanical systems, smaller volume of functional devices by one-dimensional $\mathrm{Si}$ structures is demanded. Interestingly, one-dimensional $\mathrm{Si}$ nanostructure can exhibit enhanced piezoresistance when the size is reduced to nanoscale, due to the quantum confinement effect as well as the large surface-area-to-volume ratio.

The enhanced piezoresistance of Si nanowires (SiNWs) with thicknesses ranging from 53 to $65 \mathrm{~nm}$ was observed by Toriyama et al. [5], and then they found an increase of the longitudinal piezoresistance by reducing the size of nanowires [6]. In Refs. 7 and 8, the piezoresistance of SiNWs increased by a factor of six, attributed to the size confinement effect, as compared with that of bulk Si. It was also reported that the resistance of SiNWs could be cut down by a factor of 10 under an ultrahigh tensile strain of 3.5\% [9]. Furthermore, a giant piezoresistance of SiNWs was obtained by He et al [10], and the piezoresistance of SiNWs with a diameter of $50 \mathrm{~nm}$ and a resistivity of $10 \Omega \cdot \mathrm{cm}$ can be more than one hundred times of that of bulk Si. On the other hand, an electrically controllable giant piezoresistance of SiNWs was obtained by applying an external electrical bias [11]. Piezo-pinch effect was proposed to explain the origin of the giant piezoresistance [12], which was in fact a result of the surface or interface states [13-15]. Nevertheless, Milne et al. considered that the giant piezoresistance was caused by the unsaturation of the conductance or mobility [16].

In order to modulate or further enhance the piezoresistive effect, various nanowire 
structures were investigated. Suspended SiNWs was demonstrated to be of an increased piezoresistance as compared with non-suspended SiNWs [17]. As for the Si quantum-well wire, piezoresistive coefficient was observed to be 5.5 times higher than that of bulk Si [18]. Some $\mathrm{Si}-\mathrm{X}(\mathrm{X}$ is an element other than $\mathrm{Si}$ ) alloy nanowires were found to have a larger piezoresistance than pure SiNWs do [19, 20]. Amongst them, Si and Ge can be combined together to form a SiGe alloy nanowire due to a small lattice mismatch of only $4 \%$ between them. For example, radial $\mathrm{Si} / \mathrm{Ge}$ core/shell heterostructure nanowires (HNWs) were studied experimentally [21, 22] as well as theoretically [23-25]. Moreover, axial Si/Ge HNWs were synthesized [26], and the strain distribution in axial bare Si/Ge HNWs along the [111] direction was investigated theoretically by using the molecular dynamics simulation [27]. Mechanical and band-structural properties of bare and hydrogen-passivated Si/Ge nanowire superlattices along the [100] direction were studied based on the first-principles calculation [28]. However, piezoresistive effect of axial Si/Ge HNWs along the [111] direction has not been studied yet. In this work, we have investigated the mechanical and piezoresistive properties of axial bare $\mathrm{Si} / \mathrm{Ge} \mathrm{HNW}$ along the [111] direction by using first-principles calculations and Boltzmann equation with the relaxation-time approximation. It is found that Young's modulus of HNWs depends significantly on the surface reconstruction. Whether or not the HNW has a semiconductor-like band structure and a significant piezoresistive effect is determined by the interface of heterostructure.

\section{Computational details}

We have performed the density functional theory (DFT) calculations by using the Vienna ab initio simulation package (VASP) [29]. Ultrasoft pseudopotentials with a plane-wave basis set are employed [30], and the exchange correlation potential is approximated by the generalized gradient approximation (GGA) using PW91 functional [31]. The kinetic energy cutoff for the plane-wave basis is $300 \mathrm{eV}$ and the reciprocal space is sampled by $1 \times 1 \times 8 \mathrm{k}$-points meshes. 
The minimum atom-to-atom distance between two adjacent nanowires is $15 \AA$, in order to mimic the single nanowire environment. The structures are optimized by using the conjugategradient algorithm, and the criterion of convergence for atomic forces is set to be $0.01 \mathrm{eV} / \AA$.

\section{Results and discussion}

Non-optimized bare HNWs are directly constructed from corresponding bulk materials with the diamond crystal structure, and 74 atoms are included in the supercell. The HNWs are surrounded by six equivalent (110) facets, and are oriented along the [111] direction. Crosssectional and side views of the non-optimized and the corresponding optimized HNWs are shown in Fig. 1. Olive, blue, and cyan balls denote Ge atoms, while yellow, red, and magenta balls denote Si atoms. All cross-sectional views of the non-optimized HNWs exhibit righthexagon configuration [see Figs. 1(a) and 1(c)]. Thus, non-optimized HNWs can be regarded as a regular right hexagonal prism. The atoms in green rectangle are referred to as one atomic layer, and thus three atomic layers are included in a supercell [Fig. 1(b)]. If there is an atomic layer in the supercell constructed by both $\mathrm{Si}$ and Ge atoms, we call this case a mixed layer (or mixed interface). Otherwise, if anyone of these three layers in the supercell is composed of either pure $\mathrm{Si}$ or pure Ge atoms, this is called a clear layer/interface. For simplicity, a bare HNW with 3-x layers $\mathrm{Si}$ and $x$ layers of Ge atom will hereafter be named as $\mathrm{Si}_{3-x} / \mathrm{Ge}_{x} \mathrm{HNW}$ $(0 \leq x \leq 3)$. Here, five cases of $\mathrm{Si}_{3-x} / \mathrm{Ge}_{x} \mathrm{HNWs}$ are calculated, namely, $\mathrm{Si}_{2.5} / \mathrm{Ge}_{0.5}, \mathrm{Si}_{2} / \mathrm{Ge}_{1}$, $\mathrm{Si}_{1.5} / \mathrm{Ge}_{1.5}, \mathrm{Si}_{1} / \mathrm{Ge}_{2}$, and $\mathrm{Si}_{0.5} / \mathrm{Ge}_{2.5}$ HNWs. It should be noted that $\mathrm{Si}_{2} / \mathrm{Ge}_{1}$ and $\mathrm{Si}_{1} / \mathrm{Ge}_{2} \mathrm{HNWs}$ have clear interfaces ( $x$ is an integer) in our calculation models, while $\mathrm{Si}_{2.5} / \mathrm{Ge}_{0.5}, \mathrm{Si}_{1.5} / \mathrm{Ge}_{1.5}$, and $\mathrm{Si}_{0.5} / \mathrm{Ge}_{2.5}$ HNWs have mixed interfaces ( $x$ is a decimal fraction).

Our HNW structures are similar to the structure in Ref. 13. The atomic positions of each bare HNWs with various supercell lengths are optimized to search structures with total energy minimum (i.e., the ground-state HNWs). The stabilities of ground-state bare HNWs are also confirmed by their formation energies, which are $-354.9,-342.6,-335.1,-326.2$, and - 
315.9 eV/cell for $\mathrm{Si}_{2.5} / \mathrm{Ge}_{0.5}, \mathrm{Si}_{2} / \mathrm{Ge}_{1}, \mathrm{Si}_{1.5} / \mathrm{Ge}_{1.5}, \mathrm{Si}_{1} / \mathrm{Ge}_{2}$, and $\mathrm{Si}_{0.5} / \mathrm{Ge}_{2.5} \mathrm{HNWs}$, respectively. The supercell length $\left(l_{0}\right)$ of the ground-state HNWs as a function of Ge atomic layers $(x)$ is shown in Fig. 2. It is found that the supercell length increases linearly with Ge atomic layers. The functional relationship between the supercell length $l_{0}$ and Ge atomic layers $x$ is $l_{0}=0.19 x+8.93(\AA)$ through linear fitting, which satisfies the Vegard's rule. Notice that the slope of 0.19 is larger than that of bulk SiGe alloy along the [111] direction (about 0.13). According to the fitting formula, the calculated supercell length is $8.93 \AA$ for bare SiNWs $(x=0)$, which is consistent with the value of $8.92 \AA$ A obtained by Cao et al [13].

Additionally, in the calculations, the uniaxial compressive strain $(\varepsilon<0)$ and tensile strain $(\varepsilon>0)$ are applied on bare HNWs by changing the supercell length from $l_{0}$ to $l$, according to $\varepsilon=\left(l_{0}-l\right) / l_{0}$. The total energy $E$ in dependence of strain $\varepsilon$ is obtained after reoptimization of strained HNWs. Consequently, Young's modulus can be calculated according to $Y=\left(\partial^{2} E / \partial \varepsilon^{2}\right) / V$, where $V$ is the volume of bare HNWs. Young's modulus as a function of Ge atomic layers is also shown in Fig. 2. It is found that all of them are smaller than their corresponding bulk values, which is attributed to the size confinement effect. Besides the case of $\mathrm{Si}_{2} / \mathrm{Ge}_{1} \mathrm{HNWs}$, the curve of Young's modulus vs $\mathrm{Ge}$ atomic layers exhibits a linear dependence. The exceptional Young's modulus for $\mathrm{Si}_{2} / \mathrm{Ge}_{1} \mathrm{HNW}$ is ascribed to its partial reconstruction characteristic [i.e., not all the six side edges of the right hexagonal prism will be reconstructed, see Fig. 1(e)]. Specifically speaking, red Si atom in the right layer of $\mathrm{Si}_{2} / \mathrm{Ge}_{1}$ HNW [Fig. 1(b)] stays there after optimization [Fig. 1(f)], indicating that surface reconstruction does not happen for it. To ensure the reliability of $\mathrm{Si}_{2} / \mathrm{Ge}_{1} \mathrm{HNW}$ structure, first-principles molecular-dynamics simulation with annealing temperature up to $875 \mathrm{~K}$ is performed and the same structure with partial reconstruction characteristic is obtained. However, for other HNWs, such as the $\mathrm{Si}_{1.5} / \mathrm{Ge}_{1.5} \mathrm{HNW}$ [Fig. 1(c)], all the side edges of the 
right hexagonal prism will be reconstructed. That is, the red Si atom in the right layer and cyan Ge atoms in the left layer [Fig. 1(d)] will move to the middle layer after optimization [Fig. 1(h)]. They recombine with blue Ge atoms and magenta $\mathrm{Si}$ atoms, respectively, to form pentagons at the side edges of the right hexagonal prism. This result implies that partial reconstruction is responsible for the small Young's modulus of the $\mathrm{Si}_{2} / \mathrm{Ge}_{1}$, as shown in Fig. 2 .

Band structures of HNWs under various strains are calculated, with the zero-strain result (i.e., ground-state HNWs) shown in Fig. 3. It is interesting to find that HNWs with mixed interfaces are of metal-like band (for comparison, band structures of pure $\mathrm{Si}$ and $\mathrm{Ge}$ NWs are also presented in Fig. 3), while those with clear interfaces are of semiconductor-like band. This is attributed to the confinement effect of carriers. It is well known that there exists a band offset between $\mathrm{Si}$ and $\mathrm{Ge}$, and thus diagram of band edges along the axis of HNWs will exhibit a quantum well structure [28]. As a result, carriers in the HNWs are confined in the quantum-well zone. Considering that HNWs with clear interfaces can be regarded as abrupt heterostructures, while those with mixed interfaces can be regarded as graded heterostructures, a larger band offset can be expected for the abrupt heterostructure as compared with the graded one. Obviously, the abrupt heterostructure (clear interface) is beneficial to the confinement of carriers (i.e., suppression of carrier transport). This explains why HNWs with clear interfaces have semiconductor-like band characteristic.

By considering the non-parabolic effect, effective masses are extracted from band structure by [13]

$$
\begin{aligned}
& \frac{1}{m^{*}}=\frac{1}{\sum_{i} n_{i}+\sum_{j} p_{j}}\left(\sum_{i} \frac{n_{i}}{m_{i}^{*}}+\sum_{j} \frac{p_{j}}{m_{j}^{*}}\right), \\
& \frac{1}{m_{i, j}^{*}}=\frac{1}{\int_{B Z} \omega_{k_{z}}\left(e^{E_{i, j}\left(k_{z}\right) / K T}+1\right)^{-1} d k_{z}} \int_{B Z} \frac{\omega_{k_{z}}\left(e^{E_{i, j}\left(k_{z}\right) / K T}+1\right)^{-1}}{m_{i, j}^{*}\left(k_{z}\right)} d k_{z},
\end{aligned}
$$




$$
\frac{1}{m_{i, j}^{*}\left(k_{z}\right)}= \pm \frac{1}{\hbar^{2}} \frac{\partial^{2} E_{i, j}\left(k_{z}\right)}{\partial k_{z}^{2}}
$$

where $m^{*}$ is the weighted average effective mass of HNWs, $m_{i, j}^{*}$ and $m_{i, j}^{*}\left(k_{z}\right)$ are the weighted average, and $k_{z}$-dependent effective masses for $i$ or $j$ band, respectively. $n_{i}\left(p_{j}\right)$ is the electron (hole) concentration at $i(j)$ band, respectively. $k_{z}$ is the wave vector along the axial direction of HNWs, $\omega_{k_{z}}$ the weight for $k_{z}, E_{i, j}\left(k_{z}\right)$ the energy for $i$ or $j$ band at the $k_{z}$ point, $B Z$ the Brillouin zone, $K$ the Boltzmann constant, $T$ the absolute temperature, and $\hbar$ the reduced Planck constant. In addition, carrier concentration can be obtained according to Fermi-Dirac statistics.

Calculated results show that both effective mass and carrier concentration for HNWs with mixed interfaces do not vary apparently with strains due to the metal-like band structure. Therefore, Table 1 presents only the weighted average effective mass, bandgap, and carrier concentration for semiconductor-like HNWs with clear interfaces (i.e., $\mathrm{Si}_{2} / \mathrm{Ge}_{1}$ and $\mathrm{Si}_{1} / \mathrm{Ge}_{2}$ ). It is found that the effective mass of $\mathrm{Si}_{2} / \mathrm{Ge}_{1} \mathrm{HNW}$ increases significantly with decrease of strain, which is ascribed to the electron transfer between energy bands. When the strain decreases, bandgap for $\mathrm{Si}_{2} / \mathrm{Ge}_{1} \mathrm{HNW}$ increases and electrons are transferred from band 2 to band 1 . Moreover, curvature of band 1 is smaller than that of band 2 for $\mathrm{Si}_{2} / \mathrm{Ge}_{1} \mathrm{HNW}$, and thus transferred electrons have larger effective masses than before transferred. On the other hand, as mentioned earlier, bandgaps for $\mathrm{Si}_{2} / \mathrm{Ge}_{1}$ and $\mathrm{Si}_{1} / \mathrm{Ge}_{2} \mathrm{HNWs}$ increase with decrease of strain. This leads to a significant decrease of carrier concentration with strain, especially for $\mathrm{Si}_{1} / \mathrm{Ge}_{2} \mathrm{HNWs}$.

Figure 4 shows the resistivity from Boltzmann equation with the relaxation-time approximation as a function of strain. Here we assume that electron and hole have the same relaxation time. It is found that resistivity of HNWs varies linearly with both tensile and 
compressive strain. According to the slope of the linear fitting, the piezoresistive coefficient can be obtained by

$$
\pi_{l}=\frac{1}{Y R_{0}} \frac{\partial R}{\partial \varepsilon}
$$

where $R_{0}$ is the resistivity of zero-strain HNWs. For $\mathrm{Si}_{0.5} / \mathrm{Ge}_{2.5} \mathrm{HNWs}$, the tensile-strain and compressive-strain piezoresistive coefficients have comparable values. However, for other HNWs, the compressive-strain piezoresistive coefficient is much larger than the tensile-strain one, which is consistent with literatures [7, 13]. For clarity, the absolute value of compressive-strain piezoresistive coefficient for all HNWs is shown in Fig. 5 with piezoresistive coefficients of pure $\mathrm{Si}$ and $\mathrm{Ge}$ for a comparison. It is obvious that the piezoresistive coefficients of $\mathrm{Si}_{3-x} / \mathrm{Ge}_{x} \mathrm{HNWs}$ with clear interfaces are much larger than those with mixed interfaces, and they are also larger than those of pure bare Si and Ge nanowires. From the data shown in Fig. 5, we can find that the piezoresistive coefficient of $\mathrm{Si}_{2} / \mathrm{Ge}_{1} \mathrm{HNW}$ can be as large as 1244 1/TPa. As is well known, the DFT calculations with GGA exchangecorrelation functional usually underestimate bandgaps of semiconductor, and thus the resistivity. On the other hand, the work by He et al. showed that piezoresistive coefficients increase with the resistivity [10]. Consequently, HNWs with clear interfaces in fact should be of larger piezoresistive coefficients than calculated values in Fig. 5. This result implies that bare $\mathrm{Si}_{3-x} / \mathrm{Ge}_{x} \mathrm{HNW}$ s with clear interfaces are promising for applications in pressure sensors.

\section{Conclusion}

In conclusion, piezoresistive properties of bare $\mathrm{Si}_{3-x} / \mathrm{Ge}_{x} \mathrm{HNWs}$ have been studied by using the DFT. It is found that the Young's modulus of HNWs depends significantly on the surface reconstructions. Partial reconstruction of the $\mathrm{Si}_{2} / \mathrm{Ge}_{1} \mathrm{HNW}$ results in a much smaller Young's modulus than those of other HNWs. Whether or not the HNW has semiconductorlike band and significant piezoresistive effect is determined by the heterostructure interface. 
$\mathrm{Si}_{3-x} / \mathrm{Ge}_{x} \mathrm{HNWs}$ with clear interfaces are of semiconductor-like band, while those with mixed interfaces are of metal-like band. The piezoresistive coefficients of $\mathrm{Si}_{3-x} / \mathrm{Ge}_{x} \mathrm{HNWs}$ with clear interfaces are much larger than those with mixed interfaces, and they are also larger than the piezoresistive coefficients of pure bare $\mathrm{Si}$ and Ge nanowires. These results indicate that bare $\mathrm{Si}_{3-x} / \mathrm{Ge}_{x} \mathrm{HNWs}$ with clear interfaces have potential applications as pressure sensors.

\section{Acknowledgments}

This work was supported by the National Natural Science Foundation of China (Nos. 61201032, 62370042, 61006061), the National Science Foundation of Jiangsu Province of China (Nos. BK20131108 and BK20151409), and the National Basic Research Program of

China (No. 2015CB352106). The authors would like to acknowledge Shanghai Supercomputer Center. 


\section{References}

[1] C.S. Smith, Phys. Rev. 94 (1954) 42.

[2] J.C. Suhling, R.C. Jaeger, IEEE Sens. J 1 (2001) 14.

[3] Y.B. Ning, Y. Loke, G. Mchinnon, Sens. Actuat. A 48 (1995) 55.

[4] F. Gretillat, M.A. Gretillat, N.F. de Rooij, J. Microelectromech. S. 8 (1999) 243.

[5] T. Toriyama, D. Funai, S. Sugiyama, J. Appl. Phys. 93 (2003) 561.

[6] T. Toriyama, Y. Tanimoto, S. Sugiyama, J. Microelectromech. S. 11 (2002) 605.

[7] K. Reck, J. Richter, O. Hansen, E.V. Thomsen, Proceedings of the 2008 IEEE conference on microelectromechanical systems (New York, USA) (2008) 717.

[8] U.K. Bhaskar, T. Pardoen, V. Passi, J.P. Raskin, Appl. Phys. Lett. 102 (2013) 031911.

[9] A. Lugstein, M. Steinmair, A. Steiger, H. Kosina, E. Bertagnolli, Nano Lett. 10 (2010) 3204.

[10] R.R. He, P.D. Yang, Nat. Nanotechnol. 1 (2006) 42.

[11] P. Neuzil, C.C. Wong, J. Reboud, Nano Lett. 10 (2010) 1248.

[12] A.C.H. Rowe, Nat. Nanotechnol. 3 (2008) 311.

[13] J.X. Cao, X.G. Gong, R.Q. Wu, Phys. Rev. B 75 (2007) 233302.

[14] T. Barwicz, L. Klein, S.J. Koester, H. Hamann, Appl. Phys. Lett. 97 (2010) 023110.

[15] T.K. Kang, Appl. Phys. Lett. 100 (2012) 163501.

[16] J.S. Milne, I. Favorskiy, A.C.H. Rowe, S. Arscott, C. Renner, Phys. Rev. Lett. 108 (2012) 256801.

[17] A. Koumela, D. Mercier, C. Dupre, G. Jourdan, C. Marcoux, E. Ollier, S.T. Purcell, Nanotechnol. 22 (2011) 395701.

[18] C. Pramanik, S. Banerjee, H. Saha, C.K. Sarkar, Nanotechnol. 17 (2006) 3209.

[19] A.C.H. Rowe, A. Donoso-Barrera, C. Renner, S. Arscott, Phys. Rev. Lett. 100 (2008) 145501. 
[20] F. Murphy-Armando, S. Fahy, Phys. Rev. B 86 (2012) 035202.

[21] L. Chen, F. Cai, U. Otuonye, W.D. Lu, Nano Lett. 16 (2016) 420.

[22] N. Fukata, M. Yu, W. Jevasuwan, T. Takei, Y. Bando, W. Wu, Z.L. Wang, ACS Nano 9 (2015) 12182.

[23] J. Kim, K.Y. Kim, H.J. Choi, K.H. Hong, J. Phys. Chem. C 118 (2014) 20710.

[24] K.D. Bi, J.Q. Wang, Y.J. Wang, J.J. Sha, Z. Wang, M.H. Chen, Y.F. Chen, Phys. Lett. A, $376(2012) 2668$.

[25] S. Huang, L. Yang, Appl. Phys. Lett. 98 (2011) 093114.

[26] H. Geaney, E. Mullane, Q.M. Ramasse, K.M. Ryan, Nano Lett. 13 (2013) 1675.

[27] J.G. Swadener, S.T. Picraux, J. Appl. Phys. 105 (2009) 044310.

[28] N. Akman, E. Durgun, S. Cahangirov, S. Ciraci, Phys. Rev. B 76 (2007) 245427.

[29] G. Kresse, J. Furhmuller, Comput. Mater. Sci. 6 (1996) 15.

[30] D. Vanderbilt, Phys. Rev. B 41 (1990) 7892.

[31] J.P. Perdew, Y. Wang, Phys. Rev. B 45 (1992) 13244. 


\begin{tabular}{ccccccc}
\hline \multicolumn{2}{l}{ Strain } & $-2.5 \%$ & $1 \%$ & $0 \%$ & $1 \%$ & $2.5 \%$ \\
\hline $\mathrm{Si}_{2} / \mathrm{Ge}_{1}$ & $m^{*}\left(\mathrm{~m}_{0}\right)$ & 1.983 & 1.457 & 1.110 & 0.799 & 0.635 \\
\cline { 2 - 6 } & $n\left(10^{20} \mathrm{~cm}^{-3}\right)$ & 0.90 & 1.02 & 1.28 & 1.74 & 2.21 \\
\hline$m^{*}\left(\mathrm{~m}_{0}\right)$ & 1.068 & 1.034 & 1.00 & 0.971 & 0.911 \\
\cline { 2 - 7 } $\mathrm{Si}_{1} / \mathrm{Ge}_{2}$ & $E_{g}\left(\mathrm{meV}^{2}\right)$ & 115.19 & 95.32 & 71.83 & 55.38 & 28.72 \\
\cline { 2 - 7 } & $n\left(10^{20} \mathrm{~cm}^{-3}\right)$ & 0.90 & 1.30 & 1.94 & 2.52 & 5.20 \\
\hline
\end{tabular}

Table 1. Effective mass, band gap, and carrier concentration of $\mathrm{Si}_{3-x} / \mathrm{Ge}_{x} \mathrm{HNWs}$ under various strains. 
Figure 1. (Color online) Non-optimized HNWs: (a) cross-section and (b) side-view of $\mathrm{Si}_{2} / \mathrm{Ge}_{1}$, (c) cross-section and (d) side-view of $\mathrm{Si}_{1.5} / \mathrm{Ge}_{1.5}$. Optimized HNWs: (e) cross-section and (f) side-view of $\mathrm{Si}_{2} / \mathrm{Ge}_{1}$, (g) cross-section and (h) side-view of $\mathrm{Si}_{1.5} / \mathrm{Ge}_{1.5}$. Olive, blue, and cyan balls denote Ge atoms, while yellow, red, and magenta balls denote Si atoms.

Figure 2. (Color online) Supercell length $l_{0}$ and Young's modulus $Y$ of ground-state $\mathrm{Si}_{3-x} / \mathrm{Ge}_{x}$ HNWs as a function of Ge layers $x$.

Figure 3. Band structure of zero-strain $\mathrm{Si}_{3-x} / \mathrm{Ge}_{x} \mathrm{HNWs}$ : (a) $\mathrm{Si}_{3}$, (b) $\mathrm{Si}_{2.5} / \mathrm{Ge}_{0.5}$, (c) $\mathrm{Si}_{2} / \mathrm{Ge}_{1}$, (d) $\mathrm{Si}_{1.5} / \mathrm{Ge}_{1.5}$, (e) $\mathrm{Si}_{1} / \mathrm{Ge}_{2}$, (f) $\mathrm{Si}_{0.5} / \mathrm{Ge}_{2.5}$ and (g) $\mathrm{Ge}_{3}$.

Figure 4. (Color online) Resistivity of $\mathrm{Si}_{3-x} / \mathrm{Ge}_{x} \mathrm{HNWs}$ as a function of strain.

Figure 5. (Color online) The absolute value of piezoresistive coefficient of $\mathrm{Si}_{3-x} / \mathrm{Ge}_{x} \mathrm{HNWs}$. 


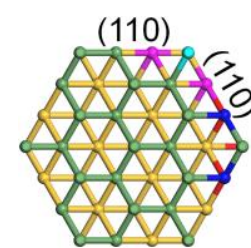

(a)

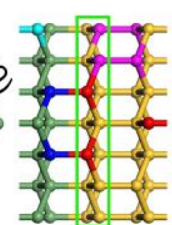

(b)

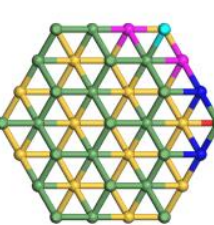

(c)

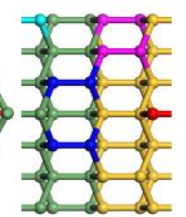

(d)

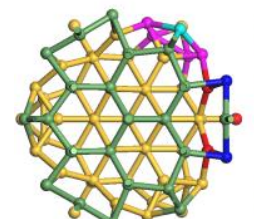

(e)

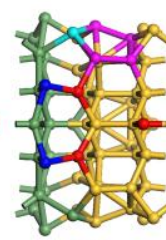

(f)

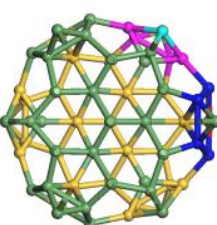

(g) (h)

Fig. 1 


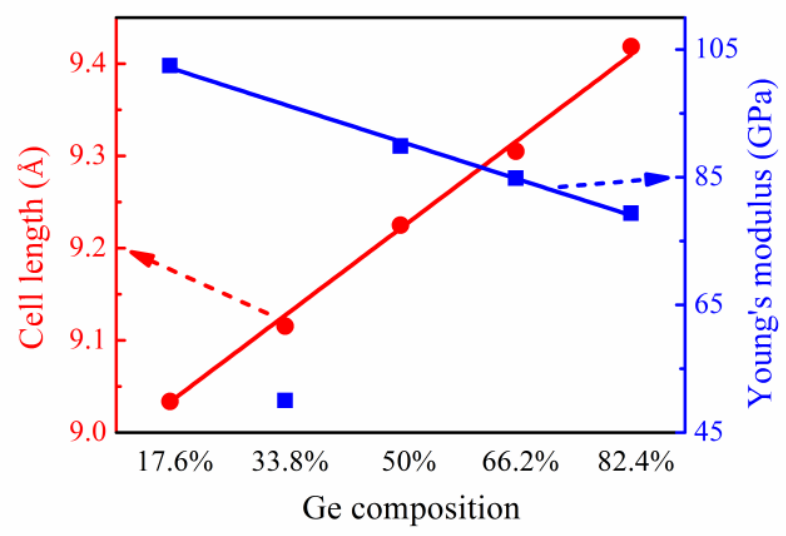

Fig. 2 


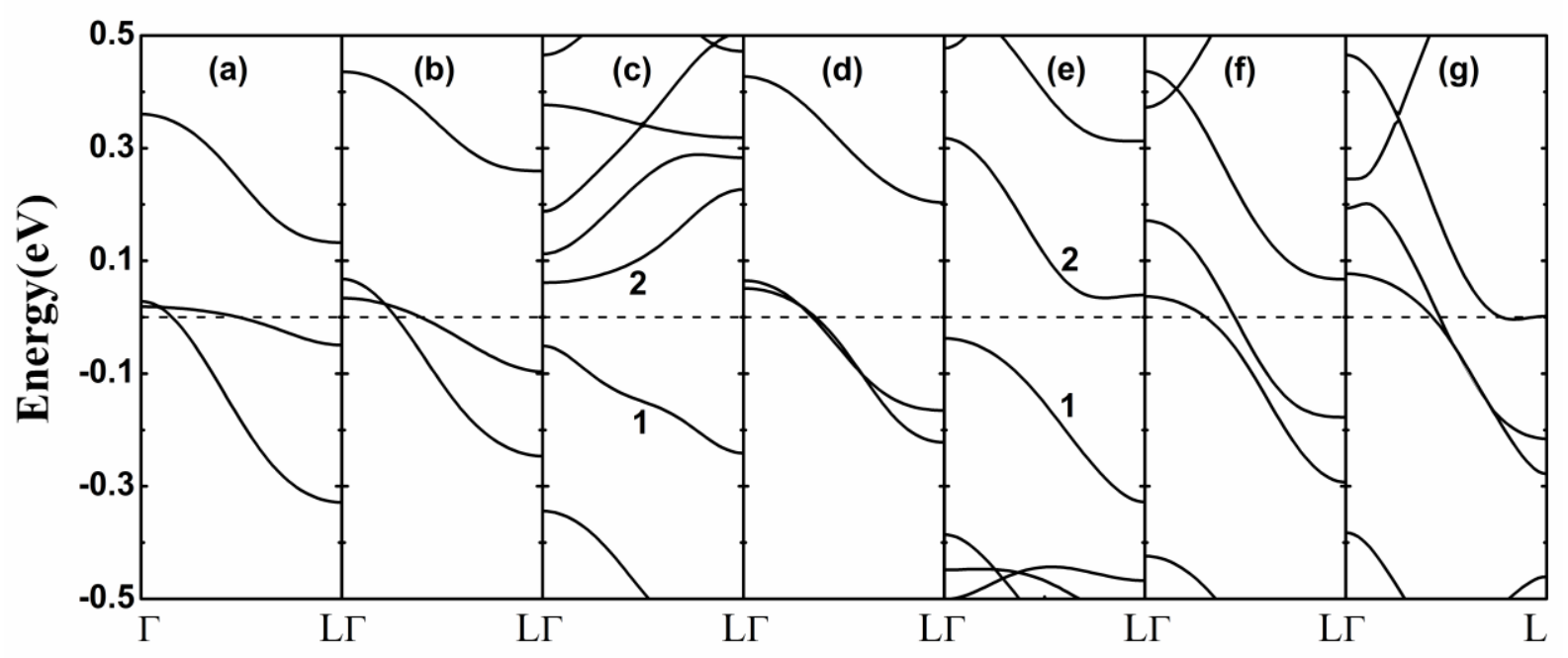

Fig. 3 


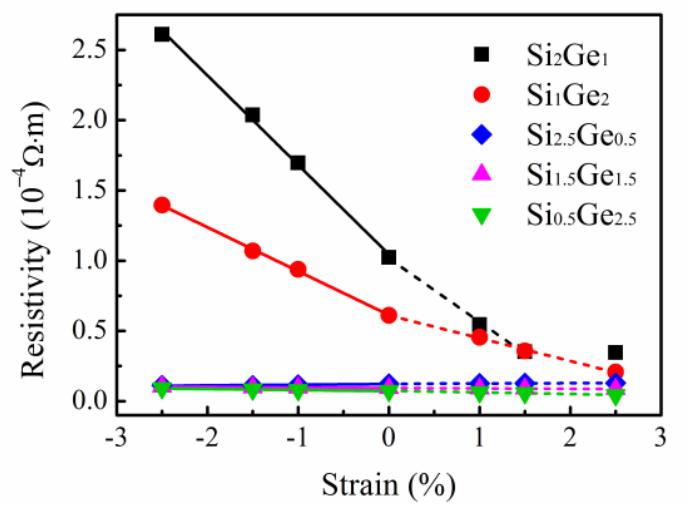

Fig. 4 


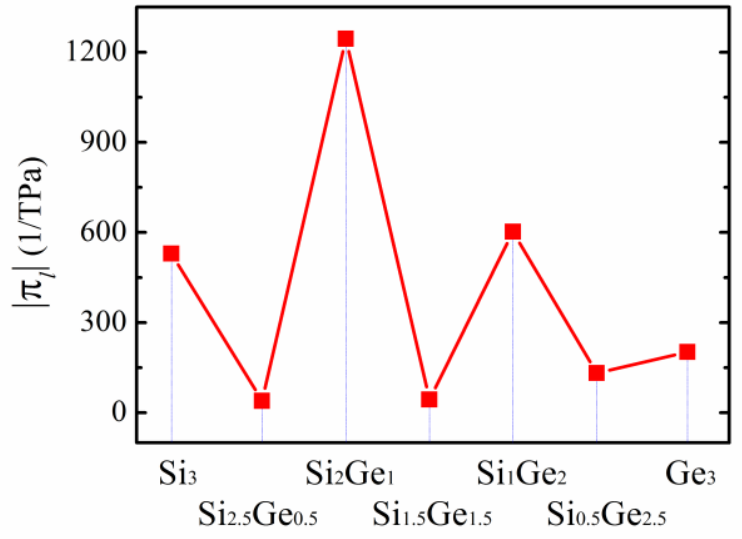

Fig. 5 